\title{
Acceso a los servicios de salud de los adultos mayores. Antioquia - Colombia
}

\author{
Access to health services for older adults. Antioquia - Colombia
}

Maria Eugenia Peña-Montoya', Maria Garzon-Duque², Doris Cardona-Arango ${ }^{3}$, Angela Segura-Cardona ${ }^{4}$

1 Enfermera. Aspirante a Magister en Epidemiologia. Medellín, Antioquia. e-mail: genia 1 1302@hotmail.com

2 Mg en Epidemiologia. Docente Universidad CES. Medellín, Antioquia. e-mail: mgarzon@ces.edu.co

3 PhD. Demografía. Mg en Salud Pública, Mg en Epidemiología. Docente Universidad CES. Medellín, Antioquia. e-mail: dcardona@ces.edu.co

$4 \quad$ PhD. Epidemiología. Mg en Epidemiología. Docente Universidad CES. Medellín, Antioquia. e-mail: asegura@ces.edu.co

\begin{abstract}
Resumen
Objetivo: Determinar el tiempo de espera para la asignación de cita con médico general, de los adultos mayores en las regiones de Antioquia en el año 2012, en función de características sociodemográficas, económicas, del estado de salud, personales, familiares y de atención en salud. Materiales y métodos: Se realizó un estudio trasversal retrospectivo con fuente secundaria de información representativa de base poblacional, tomando como población de referencia 4.215 encuestas realizadas a adultos mayores. Se realizó análisis univariado, bivariado y se identificaron los factores que más explicaban el acceso y uso de los servicios de salud por parte de los adultos mayores. Resultados: Con respecto a las variables y su asociación con el tiempo de espera para la asignación de cita con médico general de cada una de las regiones se encontraron con significancia estadística los siguientes factores: área rural de residencia, situación de salud, no tener educación certificada o contar solo con estudios de básica primara, vivir en bajos estratos y estar afiliado al régimen. Conclusión: El tiempo de espera para la asignación de cita con médico general, aun se comporta como una barrera en el acceso a los servicios de salud de los adultos mayores del departamento de Antioquia, explicado por características tales como área de residencia, percepción del estado de salud, nivel educativo, y tipo de afiliación, esto según la región del departamento.
\end{abstract}

Palabras clave: Adulto mayor; accesibilidad a los servicios de salud; consulta y referencia. (Fuente: DeCS, Bireme).

\begin{abstract}
Objective: To determine the waiting time for the allocation of medical appointments with general practitioner for older adults over the Antioquia regions in 2012, according to their socio demographic, economic, health status, personal, family and health service characteristics. Material and methods: A transversal retrospective research was done with secondary source of representative demographic based data, taking as reference population 4.215 surveys of older adults. Univariate and bivariate analysis were performed and the factors that most explained the access and the use of health services by older adults were identified. Results: According to the described variables and its association with the waiting time for the allocation of medical appointments with general practitioner at every region, it is possible to identify the following factors with statistical significance: rural area of residence, health situation, not having certified education or only having studies in primary school, living in low strata and being affiliated to the regime. Conclusion: The waiting time for the allocation of medical appointments with general practitioner is still an
\end{abstract}


access barrier to health services for older adults all over the analyzed Antioquia regions, which is explained by features like resident area, health state perception, educational level and type of affiliation, according to the region of the department.

Keywords: Older adult; health services accessibility; referral and consultation. (Source: DeCS, Bireme).

\section{Introducción}

El Departamento de Antioquia está dividido, en términos geográficos, en nueve regiones, las cuales cuentan con características únicas en cuanto a sus comportamientos políticos, ambientales y sociales, sin embargo, en la presentaciones de los resultados se evidenciará como Medellín fue analizada de manera individual con el objetivo de mostrar de manera separada los resultados de las otras regiones del Valle de Aburrá, diferenciando así sus particularidades.

Durante el censo realizado en el años 2005, el DANE reportó en total 3'721.943 adultos mayores en todo el territorio colombiano y para ese mismo año el total de la población era de 41'468.384 personas $^{1}$, que significa que el $9 \%$ del total de la población de aquel entonces en Colombia, eran personas mayores de 60 años o más, y de éste porcentaje, el 63,12\% se encontraba concentrado en Boyacá, Tolima, Bogotá, Cundinamarca, Caldas, Valle del Cauca, Quindío, Santander, Atlántico, Bolívar y Antioquia; adicionalmente, el $28 \%$ se encuentra en las principales ciudades: Bogotá, Medellín, Cali y Barranquilla.

Las poblaciones sufren grandes trasformaciones dentro de sus roles, creencias y políticas, es así como el mundo en desarrollo está en una continua transformación en donde "el envejecimiento es un fenómeno que se evidencia con el aumento de las personas mayores frente a la población total" 2 .

Según Gallegos y colaboradores 3 , en los años por venir, las principales dificultades que se derivan del envejecimiento tienen que ver con el impacto financiero de la institución, la necesidad de asignar a las pensiones jubilaciones y fondos para

un gran envejecimiento, así como para proporcionar la amplia variedad de servicios de preventivo, asistencial, y servicios de salud curativos requeridos por personas de edad avanzada.

Es de anotar que sólo a partir del año 2008 con la Ley $1251^{4}$, se le exigió al Ministerio de Protección Social definir y desarrollar una Política Nacional de Envejecimiento y Vejez, y es a partir de allí que se impulsa con más fuerza la construcción de estrategias encaminadas a mejorar la calidad de vida de las persona mayores de 60 años ${ }^{5}$; según esta ley: un adulto mayor es toda persona con edad igual o mayor de 60 años. Las diferentes culturas en el mundo, han conocido y desarrollado este concepto. Sin embargo, en términos generales, se trata de un ciclo vital afectado por múltiples factores ${ }^{6}$, tanto culturales como sociales, económicos, políticos y de salud, entre los que no se puede olvidar: la seguridad social recordando que los adultos mayores solicitan diferentes atenciones y profesionales de la salud, como consecuencia de las enfermedades crónicas desarrolladas durante toda su vida, un hecho indiscutible es que la población anciana es una de las mayores usuarias de los servicios de salud 7 .

El termino acceso, está definido como la facilidad que tiene una persona de utilizar un servicio en el momento que lo necesita ${ }^{8}$. Para el doctor Hipólito Pabón", "la accesibilidad es la condición variable de la población de poder utilizar o ser atendido por los servicios de salud", enmarcada en aspectos geográficos, económicos, organizacionales y de disponibilidad.

El acceso a los servicios de salud está caracterizado por diferentes factores, dentro de los cuales se pueden identificar aquellos socioeconómicos 10 , que incluyen factores personales, familiares, sociales, del estado de salud, financieros y de atención en salud; y es por eso que para cierta población, su capacidad adquisitiva limita el acceso a los servicios de salud, de igual manera se presentan barreras 
económicas para la consecución de medicamentos ${ }^{9}$.

Según Blanco ${ }^{11}$, las organizaciones internas de los servicios en ocasiones, establece barreras de tiempo a los pacientes que han logrado llegar a la institución, enmarcado en largas colas y Pabón", indica la poca disponibilidad de los recursos hace del tiempo de espera entre la solicitud de una consulta especializada y la fecha de consulta, un determinante en el acceso a los servicios de salud.

Para el presente estudio se analizó el acceso de los servicios de salud, de la población adulta mayor del departamento de Antioquia, a través de una de sus barreras, la de disponibilidad, mediante la variable tiempo que esperan los adultos mayores en Antioquia para la asignación de cita con médico general; según la Circular Externa 056 del 200912, la entidades prestadoras de salud deben asignar las citas solicitadas por quienes la requieran en un tiempo no mayor de cinco días.

\section{Materiales y métodos}

Se diseñó un estudio cuantitativo, tipo observacional transversal con fuente secundaria de información; se trabajó con el total de la información obtenido en el proyecto macro "Situación de salud y condiciones de vida del adulto mayor, Departamento de Antioquia, 2012 "13, por tal razón para el presente estudio no se realizó cálculos del tamaño de la muestra, sin embargo, para desarrollar el estudio macro, del que se deriva la presente investigación se utilizó la fórmula de Fleiss en poblaciones finitas, estimada para una población de 671.590 adultos mayores $^{14}$, con un nivel de confianza del 95\%, un error de muestreo del 5\%, con una proporción de adultos mayores con buen estado de salud del $50 \%$, y un efecto de diseño (deff) del 1,0. La muestra fue ampliada en un $10 \%$ para corregir posibles pérdidas de información, quedando constituida la muestra en 4.215 adultos mayores; además se empleó un muestreo polietápico. Se incluyeron los registros de interés para el estudio con el 70\% o más de los datos diligenciados; por su parte se excluyeron aquellos registros que tuvieron errores en el diligenciamiento.
El control de sesgos estuvo supeditado a la inspección de los datos que se había realizado previamente desde el estudio macro; sin embargo, para realizar el presente estudio se procedió a realizar, nuevamente, la revisión de la información de las variables de interés.

Para evitar incluir variables de confusión se realizó una revisión de literatura con el fin de cruzar y verificar información sobre el área de estudio, además se realizaron pruebas estadísticas como la regresión logística múltiple para ajustar las variables que se presentaran como de confusión ${ }^{15}$.

La información fue analizada con la ayuda del paquete estadístico SPSS versión 21, licencia de la Universidad CES, y el programa Epidat 4,1.

Para el análisis univariado, se describieron variables personales, sociales, familiares, económicas, del estado de salud y de la atención en salud, analizadas bajo cálculos estadísticos tales como distribuciones de frecuencia $y$ porcentuales, así como también estadísticos descriptivos para aquellas variables cuantitativas, además se trabajó con factores de expansión calculados de acuerdo a la proyección poblacional del DANE de adultos mayores para el departamento de Antioquia en el segundo semestre del 2012 y la inferencia poblacional se realizó con la ayuda de los datos obtenidos de las personas que fueron encuestadas; de esta manera, para el análisis univariado se trabajó con un $\mathrm{N}=671.590$.

Para el análisis bivariado se utilizaron las pruebas estadísticas, a saber: chi ${ }^{2}$, prueba exacta de Fisher y regresión simple para las variables explicativas politómicas; además de contar con sus respectivas prevalencia de riesgo (RP), todas las pruebas se calcularon con una confianza del 95\% y un error del 5, lo que equivale a un $\mathrm{p}<0.05$; las variables cuantitativas fueron recategorizadas para realizar el análisis bivariado y multivariado.

El tiempo de espera para la asignación de cita con médico general: según la circular externa 0056 de $2009^{12}$, no debe ser superior de cinco días, razón por la cual para el presente estudio se tomó como 
un tiempo aceptable el que era menor o igual a cinco días e inaceptable todo tiempo mayor de este número de días, dejando así dicotómica la variable desenlace para los análisis bivariado y multivariados.

Para el análisis multivaraido, se trabajó con la Regresión Logística Múltiple, la variable que se tomó como dependiente fue el tiempo de espera para la asignación de cita con médico general, y como independientes aquellas que en los análisis bivariados, según región, presentaron un valor de $\mathrm{p}<0,25$ (Criterio de Hosmer y Lemeshow) y aquellas que por decisión de los investigadores se consideraron necesaria para aportar a la explicación.

\section{Resultados}

En cuanto a las características sociodemográficas de los adultos mayores de Antioquia, se pudo observar que Medellín cuenta con la mayor cantidad de adultos mayores $(52,8 \%)$, en relación a Antioquia y el menor número de éstos, se encuentran ubicados en el Nordeste $(2,4 \%)$. Con respecto a la edad, la gran mayoría de los adultos mayores de Antioquia, tienen edades entre los 60 y 74 años, mientras que sólo el $0.1 \%$ de ellos tienen 100 años o más; las regiones que albergan adultos mayores centenarios son Bajo Cauca, Nordeste, Norte y Suroeste; el 72,8\% de la población adulta fue del sexo femenino y más del $90 \%$ de la población adulta mayor antioqueña reside en el área urbana, el $43 \%$ de los adultos mayores del departamento reportó estar casado o en unión libre. También se observa que en su mayoría no habían terminado la primaria $(39,4 \%)$, con predominio de esta tendencia en las regiones de Bajo Cauca y Urabá, por su parte la ciudad de Medellín alberga el mayor porcentaje de población que realizó estudios universitarios.

Se pudo observar que el $54,7 \%$ de los adultos mayores al momento de la encuesta, vivían en estratos 0,1 y 2 y el $0,1 \%$ de ésta población en estrato 5. Las regiones que muestran tener mayor proporción de personas en estratos 0 y 1 son: Bajo Cauca y Magdalena Medio, en la ciudad de Medellín se evidencia adultos mayores que residen en estratos 4 y 5 ; según los datos del estudio, la mitad de los adultos mayores no contaban con ingresos en el mes anterior a la encuesta, siendo Oriente y Bajo Cauca las regiones en donde se presentó un mayor porcentaje de adultos mayores que refirieron no haber obtenido algún ingreso en el mes inmediatamente anterior.

Al indagar por la afiliación al Sistema General de Seguridad Social en Salud (SGSSS) se encontró que el 95,1\% de los adultos mayores de Antioquia contaba con esta afiliación, distribuidos de la siguiente manera: la mitad hacían parte del régimen contributivo y sólo el $0,2 \%$ se encontraban en regímenes especiales.

En cuanto a la percepción que tiene los adultos mayores de las diferentes regiones de Antioquia, sobre sus relaciones sociales, en términos generales, se pudo identificar una tendencia a percibirlas como buenas, sólo en el Norte se evidencia una mayor tendencia de ésta percepción entre mala y regular: 35,2\%.

La percepción que tienen los adultos mayores en la atención de salud es un tema sinérgico en la identificación de servicios de calidad y que en ocasiones va ligado de las misma percepción de salud; el 57,8\% de los adultos mayores del departamento de Antioquia expresan tener una buena atención en salud; la población del Bajo Cauca, Magdalena Medio, Oriente, Medellín y Valle de Aburrá fueron las regiones que en mayor medida respondieron percibir una atención entre mala y regular (22\% de adultos de cada una de estas poblaciones).

Al indagar por hospitalización reciente (en los últimos cuatro meses) se encontró que le 7,4\% de la población de adultos mayores del departamento de Antioquia refirió haber requerido de éste servicio y más de la mitad de los adultos mayores de cada una de las regiones requirieron de consultas con médico general.

Al revisar la información obtenido sobre el tiempo que esperan los adultos mayores para la asignación de cita con médico general, se pudo identificar que en la mitad y menos de esta población reportaron esperar periodos de tiempo que van desde 25 minutos (Urabá: Me: 25 minutos 
Ri:23,66 horas) hasta 5 días (Valle del Aburra Me: 5 días Ri: 28 días), sin embargo, como se puede observar estos datos muestran rangos intercuartilicos(Ri) que pasan hasta por los 28 días de espera, tal es el caso en las siguientes regiones: Bajo Cauca Me: 2 días Ri: 8 días, Oriente Me: 2 días Ri: 7 días, Valle del Aburra (antes mencionada), Occidente Me: 2 horas Ri: 7,9 días y el Suroeste Me: 6 horas Ri: 19,9 días siendo la moda de esta región 20 días. Con respecto a las variables descritas y su asociación con el tiempo de espera para la asignación de cita con médico general de cada una de las regiones se pudo identificar los siguientes factores de riesgo: en la ciudad de Medellín el área de residencia rural (IC: 1,018 - 8,990 ), en la región del Oriente, percibir la situación de salud como buena (IC: 1,579 8,186 ), y en la región del Suroeste, vivir en el área rural (IC: 5,934 - 174,499), no tener educación certificada (IC: 1,697 - 23,194), haber realizado la primaria (IC: 1,732 - 10,274) en comparación con quienes realizaron estudios superiores, vivir en estratos 0 y 1 (IC: 1,998 - 47,863), además del 2 (IC: 3,492 - 16,019) en comparación con los estratos 4 y 5 , estar afiliado al régimen subsidiado (IC: 2,449 - 15,875) en comparación con los adultos mayores que estaban afiliados al régimen subsidiado.

Por otro lado, los factores protectores que fueron identificados en este análisis por regiones, se comportaron de la siguiente manera: en la ciudad de Medellín, vivir en estratos 1 (IC: 0,019 - 0,915), 2 (IC: 0,028 - 0,832) y 3 (IC: 0,031 - 0,997) en comparación con quienes residen en estratos $4 \mathrm{y}$ 5; en la región del Nordeste ser mujer (IC: 0,107 0,930 ) en comparación con ser hombre; en la región del Occidente, vivir en estratos 0 y 1 (IC: $0,052-0,458$ ), y en el estrato 2 (IC: $0,118-1,038$ ) en comparación con los estratos 3 y 4, al igual que en Urabá, en los estratos 0 y 1 (IC: 0,090 - 0,482) y en el Valle de Aburra, estar soltero (IC: 0,264 0,935 ) en comparación con quienes reportaron vivir con su pareja sentimental. Ver tablas 1- 10.

Tabla 1. Características de los adultos mayores que más aportan a la explicación del tiempo de espera para la asignación de cita con médico general. Región de Bajo Cauca- Antioquia, 2012

\begin{tabular}{|c|c|c|c|c|c|}
\hline & bles & $\begin{array}{c}\text { RP } \\
\text { Crudo }\end{array}$ & IC: $95 \%$ & RP ajustado & (IC: $95 \%$ ) \\
\hline Edad & $\begin{array}{l}\leq 74 \text { años } \\
\geq 75 \text { años }\end{array}$ & 1,239 & $0,647-2,371$ & 0,961 & $0,468-1,975$ \\
\hline Área de residencia & $\begin{array}{l}\text { Rural } \\
\text { Urbana }\end{array}$ & 0,236 & $0,030-1,845$ & 0,158 & $0,018-1,385$ \\
\hline & 1 & 1,405 & $0,402-4,907$ & 1,691 & $0,436-6,559$ \\
\hline Estrato socioeconómico & 2 & 2,423 & $0,634-9,259$ & 2,725 & $0,661-11,242$ \\
\hline & 3 & 1 & - & 1 & - \\
\hline Estado civil & $\begin{array}{l}\text { Soltero } \\
\text { Con pareja sentimental }\end{array}$ & 0,798 & $0,437-1,457$ & 0,756 & $0,373-1,531$ \\
\hline Tipo de afiliación & Subsidiado & 1,17 & $0,538-2,547$ & 1,075 & $0,453-2,552$ \\
\hline
\end{tabular}


Tabla 2. Características de los adultos mayores que más aportan a la explicación del tiempo de espera para la asignación de cita con médico general. Región del Magdalena Medio

\begin{tabular}{|c|c|c|c|c|c|}
\hline \multicolumn{2}{|c|}{ Variables } & $\mathbf{R P}$ & IC: $95 \%$ & RP ajustado & (IC: 95\%) \\
\hline Edad & $\begin{array}{l}\leq 74 \text { años } \\
\geq 75 \text { años }\end{array}$ & 1,359 & $0,428-4,316$ & 1,327 & $0,411-4,285$ \\
\hline Sexo & $\begin{array}{l}\text { Mujer } \\
\text { Hombre }\end{array}$ & 1,367 & $0,485-3,850$ & 1,291 & $0,440-3,784$ \\
\hline Estado civil & $\begin{array}{l}\text { Soltero } \\
\text { Casado }\end{array}$ & 1,179 & $0,429-3,242$ & 1,217 & $0,416-3,558$ \\
\hline Tipo de afiliación & Subsidiado & 0,815 & $0,275-2,417$ & 0,795 & $0,264-2,401$ \\
\hline
\end{tabular}

Tabla 3. Características de los adultos mayores que más aportan a la explicación del tiempo de espera para la asignación de cita con médico general. Región Ciudad de Medellín

\begin{tabular}{|c|c|c|c|c|c|}
\hline \multicolumn{2}{|c|}{ Variables } & \multirow{2}{*}{$\begin{array}{c}\begin{array}{c}\text { RP } \\
\text { Crudo }\end{array} \\
3,819\end{array}$} & \multirow{2}{*}{$\begin{array}{c}\text { IC: } \mathbf{9 5 \%} \\
1,468-9,937\end{array}$} & \multirow{2}{*}{$\begin{array}{r}\text { RP ajustado } \\
3,025\end{array}$} & \multirow{2}{*}{$\begin{array}{l}\text { (IC: } 95 \%) \\
1,018-8,990\end{array}$} \\
\hline Área de residencia & $\begin{array}{l}\text { Rural } \\
\text { Urbano }\end{array}$ & & & & \\
\hline \multirow{4}{*}{ Nivel de escolaridad } & Sin educación & 0,319 & $0,060-1,693$ & 1,583 & $0,177-14,161$ \\
\hline & Primaria & 0,203 & $0,045-0,914$ & 1,045 & $0,139-7,852$ \\
\hline & Segundaria & 1,019 & $0,189-5,500$ & 3,244 & $0,391-26,881$ \\
\hline & Superior & 1 & 1 & 1 & 1 \\
\hline \multirow{4}{*}{ Estrato socioeconómico } & 1 & 0,049 & $0,010-0,252$ & 0,133 & $0,019-0,915$ \\
\hline & 2 & 0,078 & $0,018-0,336$ & 0,152 & $0,028-0,832$ \\
\hline & 3 & 0,105 & $0,023-0,489$ & 0,175 & $0,031-0,997$ \\
\hline & 4 y 5 & 1 & 1 & 1 & 1 \\
\hline Estado civil & $\begin{array}{l}\text { Soltero } \\
\text { Casado }\end{array}$ & 0,724 & $0,407-1,286$ & 0,684 & $0,333-1,406$ \\
\hline Tipo de afiliación & $\begin{array}{l}\text { Subsidiado } \\
\text { Contributivo }\end{array}$ & 1,329 & $0,735-2,403$ & 0,749 & $0,363-1,545$ \\
\hline Ingresos económicos & $\begin{array}{l}\text { No } \\
\mathrm{Si}\end{array}$ & 1,801 & $1,011-3,211$ & 1,268 & $1,268-2,650$ \\
\hline \multirow{3}{*}{ Percepción del estado de salud } & Entre malo y regular & 2,217 & $0,897-5,476$ & 1,654 & $0,477-5,733$ \\
\hline & Bueno & 2,511 & $1,410-4,472$ & 1,521 & $0,709-3,260$ \\
\hline & Entre Muy bueno y excelente & 1 & 1 & 1 & 1 \\
\hline
\end{tabular}


Tabla 4. Características de los adultos mayores que más aportan a la explicación del tiempo de espera para la asignación de cita con médico general. Región del Nordeste

\begin{tabular}{|c|c|c|c|c|c|}
\hline & Variables & $\begin{array}{c}\text { RP } \\
\text { Crudo }\end{array}$ & IC: $95 \%$ & RP ajustado & (IC: 95\%) \\
\hline Edad & $\begin{array}{l}\leq 74 \text { años } \\
\geq 75 \text { años }\end{array}$ & 1,215 & $0,444-3,328$ & 1,697 & $0,530-5,429$ \\
\hline \multirow[t]{2}{*}{ Sexo } & $\begin{array}{l}\text { Mujer } \\
\text { Hombre }\end{array}$ & 0,301 & $0,113-0,800$ & 0,316 & $0,107-0,930$ \\
\hline & Sin educación & 5,75 & $0,325-101,584$ & 1,477 & $0,066-33,249$ \\
\hline \multirow{2}{*}{ Nivel de escolaridad } & Primaria & 2,135 & $0,247-18,495$ & 0,577 & $0,053-6,343$ \\
\hline & Segundaria & 1,542 & $0,141-16,803$ & 0,803 & $0,065-9,844$ \\
\hline \multirow{4}{*}{ Estrato socioeconómico } & Superior & 1 & 1 & 1 & 1 \\
\hline & 1 & 1,776 & $0,350-9,018$ & 1,44 & $0,250-8,303$ \\
\hline & 2 & 2,24 & $0,803-6,251$ & 2,548 & $0,783-8,291$ \\
\hline & 3 y 4 & 1 & 1 & 1 & 1 \\
\hline Estado civil & $\begin{array}{l}\text { Soltero } \\
\text { Con pareja sentimental }\end{array}$ & 0,615 & $0,236-1,603$ & 0,986 & $0,327-2,973$ \\
\hline Tipo de afiliación & $\begin{array}{l}\text { Subsidiado } \\
\text { Contributivo }\end{array}$ & 0,241 & $0,088-0,662$ & 0,338 & $0,108-1,059$ \\
\hline
\end{tabular}

Tabla 5. Características de los adultos mayores que más aportan a la explicación del tiempo de espera para la asignación de cita con médico general. Región del Norte

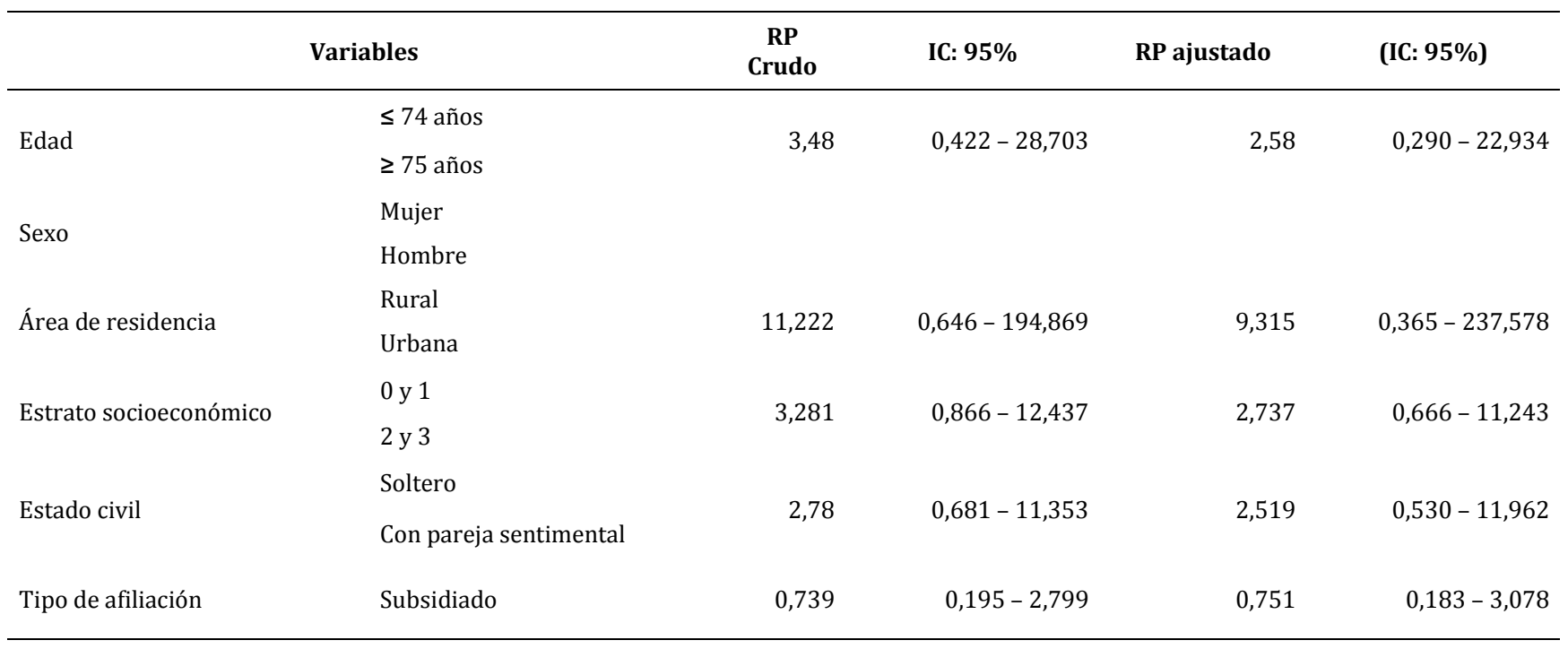


Tabla 6. Características de los adultos mayores que más aportan a la explicación del tiempo de espera para la asignación de cita con médico general. Región del Occidente

\begin{tabular}{|c|c|c|c|c|c|}
\hline \multicolumn{2}{|c|}{ Variables } & \multirow{2}{*}{$\begin{array}{c}\begin{array}{c}\text { RP } \\
\text { Crudo }\end{array} \\
1,118\end{array}$} & \multirow{2}{*}{$\begin{array}{l}\text { IC: } \mathbf{9 5 \%} \\
0,648-2,158\end{array}$} & \multirow{2}{*}{$\begin{array}{r}\text { RP ajustado } \\
1,439\end{array}$} & \multirow{2}{*}{$\begin{array}{l}\text { (IC: } 95 \%) \\
0,743-2,786\end{array}$} \\
\hline Edad & $\leq 74$ años & & & & \\
\hline Sexo & $\begin{array}{l}\text { Mujer } \\
\text { Hombre }\end{array}$ & 0,909 & $0,496-1,665$ & 0,757 & $0,369-1,553$ \\
\hline & Sin educación & 0,373 & $0,124-1,118$ & 0,512 & $0,136-1,931$ \\
\hline Nivel de escolaridad & Primaria & 0,441 & $0,180-1,081$ & 0,448 & $0,149-1,353$ \\
\hline & Segundaria y/o Superior & 1 & 1 & 1 & 1 \\
\hline & 0 y 1 & 0,158 & $0,061-0,414$ & 0,154 & $0,052-0,458$ \\
\hline Estrato socioeconómico & 2 & 0,347 & $0,131-0,919$ & 0,351 & $0,118-1,038$ \\
\hline & 3 y 4 & 1 & 1 & 1 & 1 \\
\hline Estado civil & $\begin{array}{l}\text { Soltero } \\
\text { Casado }\end{array}$ & 1,091 & $0,626-1,901$ & 1,075 & $0,555-2,083$ \\
\hline Tipo de afiliación & $\begin{array}{l}\text { Subsidiado } \\
\text { Contributivo }\end{array}$ & 1,876 & $1,004-3,503$ & 1,073 & $0,503-2,289$ \\
\hline
\end{tabular}

Tabla 7. Características de los adultos mayores que más aportan a la explicación del tiempo de espera para la asignación de cita con médico general. Región del Oriente

\begin{tabular}{|c|c|c|c|c|c|}
\hline \multicolumn{2}{|c|}{ Variables } & \multirow{2}{*}{$\begin{array}{c}\begin{array}{c}\text { RP } \\
\text { Crudo }\end{array} \\
0,908\end{array}$} & \multirow{2}{*}{$\begin{array}{c}\text { IC: } \mathbf{9 5 \%} \\
0,423-1,949\end{array}$} & \multirow{2}{*}{$\begin{array}{r}\text { RP ajustado } \\
1,29\end{array}$} & \multirow{2}{*}{$\begin{array}{l}\text { (IC: } 95 \%) \\
0,538-3,094\end{array}$} \\
\hline Edad & $\begin{array}{l}\leq 74 \text { años } \\
\geq 75 \text { años }\end{array}$ & & & & \\
\hline Sexo & $\begin{array}{l}\text { Mujer } \\
\text { Hombre }\end{array}$ & 1,503 & $0,741-3,048$ & 1,426 & $0,644-3,155$ \\
\hline Área de residencia & $\begin{array}{l}\text { Rural } \\
\text { Urbana }\end{array}$ & 2,01 & $0,783-5,158$ & 1,481 & $0,496-4,425$ \\
\hline \multirow{3}{*}{ Estrato socioeconómico } & 1 & 0,517 & $0,147-1,813$ & 0,743 & $0,159-3,483$ \\
\hline & 2 & 0,617 & $0,294-1,291$ & 0,579 & $0,252-1,330$ \\
\hline & 3 & 1 & 1 & 1 & 1 \\
\hline Estado civil & $\begin{array}{l}\text { Soltero } \\
\text { Con pareja sentimental }\end{array}$ & 1,528 & $0,771-3.029$ & 1,427 & $0,665-3,064$ \\
\hline Tipo de afiliación & $\begin{array}{l}\text { Subsidiado } \\
\text { Contributivo }\end{array}$ & 1,703 & $0,815-3,557$ & 1,193 & $0,524-2,713$ \\
\hline \multirow{3}{*}{ Percepción del estado de salud } & Entre malo y regular & 1,612 & $0,476-5,461$ & 1,353 & $0,323-5,671$ \\
\hline & Bueno & 3,029 & $1,567-5,853$ & 3,595 & $1,579-8,186$ \\
\hline & Entre Muy bueno y excelente & 1 & 1 & 1 & 1 \\
\hline
\end{tabular}


Tabla 8. Características de los adultos mayores que más aportan a la explicación del tiempo de espera para la asignación de cita con médico general. Región del Suroeste

\begin{tabular}{|c|c|c|c|c|c|}
\hline \multicolumn{2}{|c|}{ Variables } & \multirow{2}{*}{$\begin{array}{c}\begin{array}{c}\text { RP } \\
\text { Crudo }\end{array} \\
1,189\end{array}$} & \multirow{2}{*}{$\begin{array}{l}\text { IC: } \mathbf{9 5 \%} \\
0,678-2,085\end{array}$} & \multirow{2}{*}{$\begin{array}{r}\text { RP ajustado } \\
0,772\end{array}$} & \multirow{2}{*}{$\begin{array}{l}\text { (IC: 95\%) } \\
0,374-1,591\end{array}$} \\
\hline Edad & $\begin{array}{l}\leq 74 \text { años } \\
\geq 75 \text { años }\end{array}$ & & & & \\
\hline Sexo & $\begin{array}{l}\text { Mujer } \\
\text { Hombre }\end{array}$ & 1,071 & $0,617-1,861$ & 0,885 & $0,436-1,794$ \\
\hline Área de residencia & $\begin{array}{l}\text { Rural } \\
\text { Urbana }\end{array}$ & 15,75 & $3,319-74,738$ & 32,178 & $5,934-174,499$ \\
\hline \multirow{3}{*}{ Nivel de escolaridad } & Sin educación & 7,224 & $2,422-21,546$ & 6,274 & $1,697-23,194$ \\
\hline & Primaria & 2,817 & $1,451-5,471$ & 4,218 & $1,732-10,274$ \\
\hline & Segundaria & 1 & 1 & 1 & 1 \\
\hline \multirow{3}{*}{ Estrato socioeconómico } & 0 у 1 & 7,246 & $1,624-32,327$ & 9,778 & $1,998-47,863$ \\
\hline & 2 & 4,298 & $2,409-7,668$ & 7,479 & $3,492-16,019$ \\
\hline & 3 & 1 & & 1 & 1 \\
\hline Estado civil & $\begin{array}{l}\text { Soltero } \\
\text { Con pareja sentimental }\end{array}$ & 0,788 & $0,466-1,331$ & 0,512 & $0,256-1,024$ \\
\hline Tipo de afiliación & $\begin{array}{l}\text { Subsidiado } \\
\text { Contributivo }\end{array}$ & 1,992 & $1,007-3,940$ & 6,236 & $2,449-15,875$ \\
\hline \multirow{3}{*}{ Percepción del estado de salud } & Entre malo y regular & 3,515 & $0,965-12,804$ & 0,311 & $0,075-1,289$ \\
\hline & Bueno & 2,797 & $1,670-4,685$ & 1,165 & $0,581-2,337$ \\
\hline & Entre Muy bueno y excelente & 1 & 1 & 1 & 1 \\
\hline
\end{tabular}

Tabla 9. Características de los adultos mayores que más aportan a la explicación del tiempo de espera para la asignación de cita con médico general. Región del Urabá

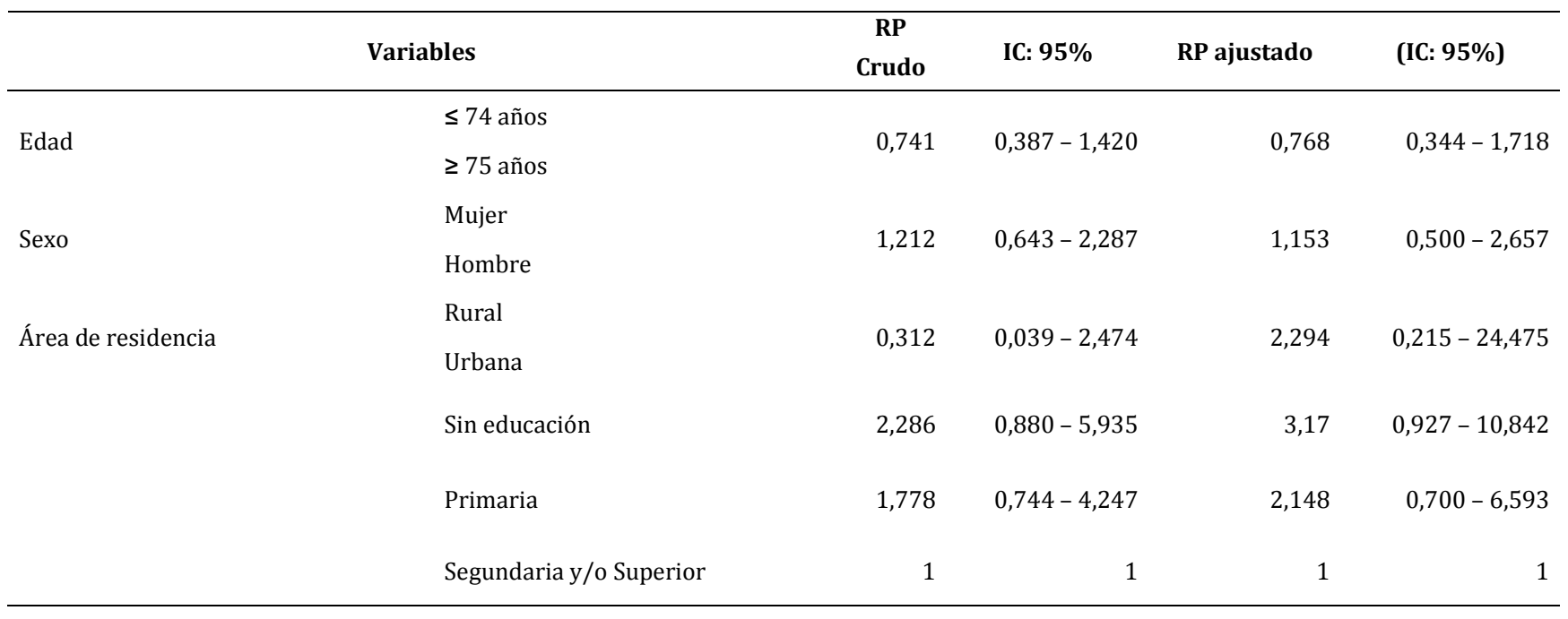




\begin{tabular}{|c|c|c|c|c|c|}
\hline \multirow{2}{*}{ Estrato socioeconómico } & 0 y 1 & \multirow{2}{*}{0,226} & \multirow{2}{*}{$0,106-0,481$} & \multirow{2}{*}{0,208} & \multirow{2}{*}{$0,090-0,482$} \\
\hline & 2,3 y 4 & & & & \\
\hline \multirow[b]{2}{*}{ Estado civil } & Soltero & \multirow[b]{2}{*}{0,702} & \multirow[b]{2}{*}{$0,376-1,311$} & \multirow[b]{2}{*}{0,692} & \multirow[b]{2}{*}{$0,312-1,536$} \\
\hline & Con pareja sentimental & & & & \\
\hline \multirow{2}{*}{ Tipo de afiliación } & Subsidiado & \multirow{2}{*}{0,417} & \multirow{2}{*}{$0,153-1,140$} & \multirow{2}{*}{0,877} & \multirow{2}{*}{$0,265-2,904$} \\
\hline & Contributivo & & & & \\
\hline \multirow{3}{*}{ Percepción del estado de salud } & Entre malo y regular & $\mathrm{NC}$ & $\mathrm{NC}$ & 0,265 & $0,011-6,152$ \\
\hline & Bueno & 1,549 & $0,854-2,812$ & 1,073 & $0,488-2,358$ \\
\hline & Entre Muy bueno y excelente & 1 & 1 & 1 & 1 \\
\hline
\end{tabular}

Tabla 10. Características de los adultos mayores que más aportan a la explicación del tiempo de espera para la asignación de cita con médico general. Región del Valle de Aburrá

\begin{tabular}{|c|c|c|c|c|c|}
\hline & Variables & $\begin{array}{c}\text { RP } \\
\text { Crudo }\end{array}$ & IC: $95 \%$ & RP ajustado & (IC: 95\%) \\
\hline Edad & $\begin{array}{l}\leq 74 \text { años } \\
\geq 75 \text { años }\end{array}$ & 1,006 & $0,537-1,882$ & 0,882 & $0,450-1,727$ \\
\hline Sexo & $\begin{array}{l}\text { Mujer } \\
\text { Hombre }\end{array}$ & 0,761 & $0,434-1,337$ & 1,013 & $0,529-1,939$ \\
\hline Estado civil & $\begin{array}{l}\text { Soltero } \\
\text { Con pareja sentimental }\end{array}$ & 0,612 & $0,356-1,052$ & 0,497 & $0,264-0,935$ \\
\hline Tipo de afiliación & $\begin{array}{l}\text { Subsidiado } \\
\text { Contributivo }\end{array}$ & 0,822 & $0,432-1,563$ & 1,172 & $0,561-2,449$ \\
\hline Ingresos & No & 0,66 & $0,385-1,134$ & 0,56 & $0,298-1,051$ \\
\hline
\end{tabular}

\section{Discusión}

El análisis del acceso a los servicios de salud, se ha venido desarrollando alrededor de importantes teorías, que van más allá de la afiliación a un régimen en salud; por su parte, el tiempo de espera para la asignación de una cita y la forma de hacerlo, es sin duda un elemento básico de los derechos relacionados con el sistema de salud y debe ser un compromiso esencial de la calidad asistencial. Las listas de espera son elementos comunes a todos los sistemas sanitarios de financiación pública, sin embargo, desde el punto de vista técnico y ético, las listas de espera no deberían incluir a ningún enfermo cuya espera pudiera comprometer su evolución o pronóstico o fueran susceptibles de generar secuelas ${ }^{16}$.

La ley colombiana, define como cinco días el tiempo máximo para la asignación de cita con médico general ${ }^{12}$, sin embargo, los tiempos de espera en la atención en salud, no han sido explorados a fondo en investigaciones, razón por la cual ha sido difícil identificar qué factores pudieran ayudar a la explicación de estos tiempos, y de esta manera en los resultado de este trabajo se evidencia problemas en el acceso a los servicios de salud, tales como, vivir en zonas rurales, percibir la salud entre mala, regular y buena, no tener educación, o niveles bajos de educación e incluso estar afiliado al régimen subsidiado, 
factores que se han identificado en las diferentes regiones de Antioquia.

Es de anotar que no se identificaron artículos de la ciudad de Medellín y las regiones del Oriente y Suroeste que hablaran de los factores riesgo en el acceso a los servicios de salud, sin embargo, se traerán a acotación algunos resultados de otras investigaciones que podrían encaminar la discusión.

En cuanto al área de residencia, identificada en la Cuidad de Medellín y en la región de Suroeste como un factor de riesgo, es importante recordar que la mayor cantidad de adultos viven en las cabeceras municipales de las regiones, sólo en la región de Occidente se evidenció un porcentaje importante $(14 \%)$ de adultos mayores que residen en el área rural, con respecto a la población de su región; siendo una posible explicación de este fenómeno que en estas regiones no se cuente con centros de salud distribuidos en zonas rurales para el alance de los adultos mayores.

Si bien, en la región del Suroeste, se identificó que los estratos socioeconómicos bajos (entre 0 y 2) se comportaron como factores de riesgo, para las regiones Occidente, Urabá y Medellín esta variable se comportó por el contrario como un factor protector; situación que podría ser explicada por las diferencias dentro del mismo departamento en las administraciones de estos servicios. Por otro lado, según la encuesta demografía y salud de 201017, alrededor de la quinta parte de los adultos mayores no han aprobado ningún año de educación, 58\% han aprobado sólo la primaria, $17 \%$ tienen secundaria y $5 \%$ tienen educación superior; situación similar se observó para el presente estudio, pues de los datos obtenidos por región, a medida que aumentaba el nivel educativo, disminuía la proporción de adultos que habían alcanzado estos niveles.

Teniendo en cuenta que el estrato socioeconómico no necesariamente define el estado económico de las personas, si determina el lugar de ubicación de las viviendas en Colombia, ya que su distribución fue determinada en una escala de menor a mayor ("de un barrio marginado a un barrio de alto nivel"), por tal motivo, con esta información se podría caracterizar el estado socioeconómico de la población; y de esta manera, según los resultados obtenido, en el Suroeste las personas más pobre son quienes se demoran más tiempo para que se les sea asignada una cita médica; por su parte en un estudio realizado en España ${ }^{18}$, donde se compararon los periodos 1997 y 1995, se estudiaron todos los mayores de 24 años, identificando que el tiempo de espera para la asignación de cita se reducía a la vez que disminuía el estado socioeconómico y a su vez, se observó que los individuos con mayor nivel educativo y socioeconómico conocían mejor el funcionamiento del sistema sanitario, y por ello presentaban mayor probabilidad de resolver problemas administrativos para acceder a los mismos; en el presente estudio regiones como Medellín, Occidente, Urabá, también demostraron una disminución del tiempo de espera para la asignación de cita en quienes vivían en estratos socioeconómicos bajos en comparación con quienes vivían en estratos más altos.

"La dependencia en la vejez se expresa de manera funcional, económica, social y psíquica, y la dependencia funcional, a su vez, en limitaciones parciales que dificultan el cuidado de sí y generan el estigma de discapacidad"19.

A su vez, en Colombia, el proceso de descentralización del sector público se inició con el Decreto 77 de 1987 y la Ley 10 de 1990, articulándose, finalmente con la reforma al Sistema de Seguridad Social en Salud en 1993, en 10 años la afiliación de la población aumentó en 10 puntos $^{20}$ y con el pasar de los años esta cobertura ha ido aumentado significativamente y los adultos mayores no han estado exentos de esta situación, según los datos obtenidos en el presente estudio la cobertura en todo el territorio antioqueño excede el $92 \%$ de población de cada una de las regiones de Antioquia, Urabá es la región en donde un porcentaje mayor de los adultos mayores no se encuentra afiliados a ningún régimen, con un 7,4\% (del total de su población), aunque son más quienes se encuentran afiliados al régimen subsidiado; por 
otro lado en Medellín y en el Valle del Aburra esta situación cambia; según Vargas y Molina en su artículo acceso a los servicios de salud en seis ciudades de Colombia ${ }^{21}$, el nuevo modelo de aseguramiento dio oportunidades a las personas de escasos recursos económicos de acceder a algunos servicios que antes no tenían, especialmente a aquellos que implican la utilización de altas tecnologías para la atención de enfermedades de alto costo.

Según un estudio realizado en Bogotá ${ }^{22}$, se evidenció inequidad en el acceso a los servicios de salud, la cual está afectando de manera importante a la población más pobre de la ciudad, y la no afiliada al Sistema General de Seguridad Social en Salud, principalmente. Para el presente estudio en la región del Suroeste Antioqueño gran parte de los adultos mayores están afiliados al sistema de salud vía régimen subsidiado y es para este tipo de afiliación que en estudios como el de Bogotá se han evidenciado mayores tiempos de espera para acceder a las citas con médico general; además se describe que el conocimiento y la falta de información se evidencia en la población de la capital de la republica estudiada, comportamiento que se relaciona con los resultado obtenidos en la región del Suroeste donde esta variables (régimen subsidiado) y otras tales como estratos socioeconómicos bajos al igual que los niveles educativos, se comportaron como factores de riesgo para acceso a los servicios de salud; por otro lado, las personas que hacen parte del régimen subsidiado deben realizar innumerables filas para acceder a una cita y en ocasiones no alcanzan fichas para que se les sea asignada.

Por otro lado, la percepción de la situación de salud, que tuvieron los adultos de las diferentes regiones, en términos generales, fue buena, muy buena y excelente, sin embargo en Magdalena Medio y Oriente no se encuestaron adultos que percibieran su salud como excelente. Bajo Cauca es la región en donde hay un mayor porcentaje de adultos que perciben su salud entre mala y regular, de hecho la Encuesta Nacional de Demografía y Salud ${ }^{23}$, explica que uno de cada 10 adultos mayores consideran excelente o buena su salud, porcentaje demasiado bajo, teniendo en cuenta que estas personas se encuentran la última etapa de la vida; de hecho en la región del Oriente esta percepción de salud entre los adultos mayores que la califican como buena se vio enmarcada como un factor de riesgo, posiblemente porque estas personas que la señalaron como buena, consultan en mayor medida que las personas que califican su salud entre muy buen y excelente, y por ello se ven enfrentadas a mayores problemas en el acceso a la salud. "La dependencia en la vejez se expresa de manera funcional, económica, social y psíquica, y la dependencia funcional, a su vez, en limitaciones parciales que dificultan el cuidado de sí y generan el estigma de discapacidad"19.

Como conclusión, el tiempo de espera para la asignación de cita con médico general, aun se comporta como una barrera en el acceso a los servicios de salud de los adultos mayores del departamento de Antioquia, explicado por características tales como área de residencia, percepción del estado de salud, nivel educativo, y tipo de afiliación, según la región del departamento. Según el artículo tiempo de espera aceptables y repercusión de la espera desde la perspectiva de los pacientes ${ }^{16}$, dentro de las consecuencias que se encontraron en los tiempos de espera prolongados para la asignación de citas, se evidencian consecuencias en las personas, tales como ansiedad, necesidad de alargar incapacidades laborales, peores pronósticos en el manejo de la enfermedad.

Como se pudo evidenciar, el presente estudio señaló algunas de las particularidades de los adultos mayores de Antioquia, dando a conocer características únicas de estos enmarcadas en el territorio de vivienda, demostrando así, la importancia de analizar la información de cada región desde sus propios resultados y no desde comportamientos globales del Departamento.

Conflicto de intereses: Ninguno declarado por autores.

\section{Referencias}

1. República de Colombia. Departamento Administrativo Nacional de Estadística. DANE. Boletín censo general 2005, perfil Medellín Antioquia [Internet]. DANE. 2006 
[citado 11 de octubre de 2013]. Recuperado a partir de: http://www.dane.gov.co/files/censo2005/PERFIL_PD F_CG2005/05001T7T000.PDF

2. Cardona-Arango D, Estrada-Restrepo A, Chavarriaga LM, Ordoñez-Molina JE, Osorio-Gomez JJ, SeguraCardona AM. Situación de salud del adulto mayor institucionalizado. Medellín: Universidad CES; 2010.

3. Gallegos-Carrillo K, Garcia-Peña C, Durán-Muñoz C, Mudgal J, Durán-Arenas L, Salmerón-Castro J. Health care utilization and health-related quality of life perception in older adults: a study of the Mexican Social Security Institute. Salud Pública México. 2008; 50(3):207-17.

4. República de Colombia. Ley 1251 de 2008. Protección, promoción y defensa de los derechos de los adultos mayores. Congreso de la República; 2008.

5. República de Colombia. Ley 1151 de 2007. Plan Nacional de Desarrollo 2006-2010. Congreso de la República; 2007.

6. Cardona-Arango D, Agudelo-García H. La flor de la vida, pensamientos en el adulto. Medellín: Universidad de Antioquia; 2006: 277.

7. Borges A, Gómez H. Uso de los servicios de salud por la población de 60 años y más en México. Rev Salud Pública México. 1998;40(1):13-23.

8. Agudelo-García HB. Hacia decisiones saludables para todos: diagnóstico de la situación de salud municipio de Envigado, 2006. Medellín: Universidad de Antioquia, Facultad Nacional de Salud Pública; 2007.

9. Pabón H. Evaluación de los servicios de salud. Conceptos, indicadores, ejemplos de análisis cuantitativo y cualitativo. Universidad el Valle. Facultad de Salud, Departamento de Medicina Social: XYZ; 1985:306.

10. Vargas-Lorenzo I, Vázquez-Navarrete ML, MogollónPérez AS. Access to health care in Colombia. Rev Salud Pública. 2010;12(5):701-12.

11. Blanco-Restrepo JH. Fundamentos de salud pública. Medellín, Colombia: Corporación para Investigaciones Biológicas; 2005.

12. República de Colombia. Superintendencia Nacional de Salud. Circular 0056 de 2009. Bogotá: Superintendencia Nacional de Salud; 2009.

13. Cardona-Arango D, Segura-Cardona A, Garzón-Duque M. Situación de salud y condiciones de vida del adulto mayor, Departamento de Antioquia, 2012. Medellín, Colombia: Editorial CES; 2013: 212.

14. República de Colombia. Departamento Administrativo Nacional de Estadística. DANE. Proyecciones de población: proyecciones municipales 2005-2011 [Internet]. 2007. Disponible en: http://dane.gov.co

15. Hernández-Sampieri R, Fernández-Collado C, BaptistaLucio P. Metodología de la investigación. México: McGraw-Hill; 2010.

16. Rodrigo I, Gabilondo L. Tiempos de espera aceptables y repercusión de la espera desde la perspectiva de los pacientes. Rev Calidad asistencial. 2007;22(4):168-79.

17. Colombia. Quinta Encuesta Nacional de Demografía y Salud. 2010. Disponible en: http://www.profamilia.org.co/.../Situacion_de_los_Adu ltos_Mayores_en_Colombia

18. Lostao L, Regidor E, Calle ME, Navarro P, Domínguez V. Evolución de las diferencias socioeconómicas en la utilización y accesibilidad de los servicios sanitarios en España entre 1987 y 1995/97. Rev Esp Salud Pública. 2001;75(2):115-28.

19. Herrera Z, Carmen M del, Marín T, Patricia B. Care transition for hospitalized dependent aged persons. Investig Educ En Enferm. 2007;25(1):40-9.

20. Escalona-Orcao AI, Diez-Cornago C. Accesibilidad geográfica de la población rural a los servicios básicos de salud: Estudio en la provincia de Teruel. Revista de estudios sobre despoblación y desarrollo rural. 2003;3: 111-150.

21. Gómez O, Monsalve S. Adulto mayor en Colombia, estadística y tendencias, Club Rotatorio, Medellín. Universidad de Antioquia; 2008: 16 p.

22. Vargas J, Molina G. Acceso a los servicios de salud en seis ciudades de Colombia: limitaciones y consecuencias. Rev Fac Nac Salud Pública. 2009;2:121-30.

23. Rubio-Mendoza ML. Equity in gaining access to health services and equity in funding being attended by them in Bogota. Rev Salud Pública. 2008;10:29-43.

24. Ojeda G, Ordóñez GM, Ochoa LH. Asociación Probienestar de la familia colombiana, Macro internacional, editores. Colombia: Encuesta Nacional de Demografía y Salud, 2010. [Bogotá] : Calverton (Md.), US: Asociación Probienestar de la Familia Colombiana, Profamilia ; ORC Macro International; 2011:1. 